\title{
MODIFIED GAUSS-LEGENDRE, LOBATTO AND RADAU CUBATURE FORMULAS FOR THE NUMEMCAL GVALUATION OF 2-D SINGULAR INTEGRALS
}

\author{
P.S. THEOCANAS \\ Department of Theoretical and Applled Mechanics \\ The National Technical University of Athens \\ 5, Heroes of Polytechnion Avenue, \\ Zographou, Athens 624, Greece \\ (Recelved June 19, 1982)
}

ABSTRACT A numerical technique, first reported in 1979 in refs.[1] and [2], for the numerical evaluation of two-dimensional Couchy-type principal-value intesrale, 1s. extended in this paper to include several cubature formulas of the Padan and Lobatto types. For the construction of such a cubature formula the 2-D elngular integral is considered as an iterated one, and the second-order pole invalved in this integral ip analyzed into a pair of complex poles, Based on this procedure, the metbods of numerical integration, valid for one-dimensional singular integrals, are extended to the case of two-dimensional singular integrals. The cubature formulas of the Lobattoand Radou-type are now formulated to include the cases where won of the destred abeciseas wa be chosen according to any appropriate criterion.

Horeover, the theory developed is enlarged to include the case of a 2-D principal-value integral, containing a logaritbaic singularity, The validity of the results is illustrated by considering certaln numerieal examples, Purthermore, a complete analysis of the convergence and the construetion of exror eatimates is also presented.

KEY WORDS AND PHRASES. Numerical integration, Numerical evaluation of $2 \rightarrow 0$ singular Integrals, Cubature Formulas of the Radau and Lobatto types, Collocation points, Error estimates for 2-D Principal Value Integrals. 1980 MATHEMATICS SUBJECT CLASSIFICATION CODES, 65032, 65030, 65699, 41A55, 


\section{INTRODUCTION}

Two-dimensional principal-value integrals are frequently encountered in engineering problems and especially in the three-diemensional theory of elasticity [3 to 5]. Yet, the methods for their numerical evaluation existing in the literature $[3,6,7]$ are not general and efficient enough to be of a wide use. One such method, developed by Cruse [4], can be considered as the basis of the well-known BoundaryIntegral Equation (BIE) technique. It should be noticed that, for the numerical evaluation of these integrals by using the BIE-method, the surface on which the integration should be performed must be discretized into infinitesimal flat pleces and the surface data of the density function should be assumed constant on each of them. On the other hand, a method based on the direct numerical integration [5,8] of the two-dimensional Cauchy principal-value integral was not generally accurate enough, thereby requiring an analytical attack on the integral.

Similar methods to the BIE-technique were proposed in refs.[7] and [8], where the numerical evaluation of two-dimensional Cauchy-type principal-value integrals on a circular region was considered, when the singularity was at the center of the circle. The results of [6] and [7] were generalized in [9] for the case of an arbitrary region.

Th1s paper presents a general method for the numerical evaluation of twodimensional principal-value integrals by constructing a cubature formula for their estimation. For the formation of such a cubature formula the two-dimensional Integral 18 considered as an fterated one, and the involved singularity is analyzed by reduction into a pair of complex singularities $[1,2]$. Based on this fact, the methods of numerical integration, developed for one-dimensional singular integrals [9 to 10], are extended to the cases of two-dimensional singular integrals by introducing a properly estimated set of collocation points.

Moreover, a typlcal integral, which was previously evaluated by Cruse [4] In a different way, 18 again determined by using the proposed numerical technique. The colncidence of the results by the two methods is satisfactory. On the other hand, the Radau and Lobatto integration rules are established for the numerical calculation of 2-D singular integrals, Several integrals, also 
a logarithmic singularity, are calculated while special attention is given to the Investigation of the convergence of the proposed method by obtaining estimate for the related error.

2. MODIFIED GAUSS-LEGENDRE CUBATURE FORMULAS.

Consider the two-dinensional singular integral $I\left(\xi_{0}, n_{0}\right)$, defined on a plane region S (Fig.1, [9]);

$$
I\left(\xi_{0}, n_{0}\right)=f_{s} w(\xi, n) \frac{f\left(\xi_{0}, n_{0}, \theta\right)}{r^{2}} u(\xi, n) d \xi d n,
$$

where:

$$
\left(\xi-\xi_{0}\right)+1\left(n-n_{0}\right)=r e^{i \theta},
$$

and $w(\xi, n)$ is a weight function of the form

$$
w(\xi, n)=w_{1}(\xi) w_{2}(n) \text {. }
$$

where the functions $w_{1}$ and $w_{2}$ are included in Table $I$.

Under the assumptions that:

1) the density function $u(\xi, n)$ of the singular integral $I\left(\xi_{0}, n_{0}\right)$ is a bounded and HoLder continuous function is $\mathrm{S}$;

11) If the surface has points at infinity, then

$u(\xi, n)=O\left(x^{-k}\right)(k>0)$ and;

111) the characteristic function $f\left(\xi_{0}, n_{0}, \theta\right)$ is bounded and for a fixed pole $x\left(\xi_{0}, n_{0}\right)$, 18 continuous with respect to $\theta$, Fricomi [11] has shown that the necessary and oufficient condition for the existence of the singular integral [2.1], in the principal value sense, is. that is characteristic $f\left(\xi_{0}, n_{0}, \theta\right)$ satisfies the condition [12]

$$
\int_{0}^{2 \pi} f\left(\xi_{0}, n_{0}, \theta\right) d \theta=0 .
$$

Let us now consider a square area $T$ of sides equal to 2 units, belonging to $S$, which surrounds the second order pole $X\left(\xi_{0}, n_{0}\right)$. Then, it is easy to show that the singular integral I becomes

$$
I\left(\xi_{0}, n_{0}\right)=\int_{S-T} w(\xi, n) \frac{f\left(\xi_{0}, n_{0}, \theta\right)}{r^{2}} u(\xi, n) d \xi d n+I_{1}\left(\xi_{0}, n_{0}\right),
$$

where 


$$
I\left(\xi_{0}, n_{0}\right)=f_{T} w(\xi, n) \frac{f\left(\xi_{0}, n_{0}, \theta\right)}{r^{2}} u(\xi, n) d \xi d n .
$$

Here, the first integral on the right-hand side of Eq.(2.5) is a regilar one, while the second (that is the integral $I_{1}$ ) must be considered in a principal-value sense.

Integrals of the form (2.6) are frequently encountered in the 3-din theory of elasticity [3], where the characteristic $f\left(\xi_{0}, n_{0}, \theta\right)$ satisfies the condition $(2,4)$. Then, according to reference [12], the following expression 1s valld:

$$
I_{1}\left(\xi_{0}, n_{0}\right)=\int_{-1}^{1} w_{1}(\xi) \frac{\left(I_{A}-I_{B}\right)}{\xi-\xi_{0}} d \xi \text {. }
$$

where:

$$
I_{A}=\int_{-1}^{1} w_{2}(n) \frac{f\left(\xi_{0}, n_{0}, \theta\right)}{n-2} u(\xi, n) d n
$$

and:

$$
I_{B}=\int_{-1}^{1} w_{2}(n) \frac{f\left(\xi_{0}, n_{0}, \theta\right)}{n-\bar{z}} u(\xi, n) d n
$$

with:

$$
z=n_{0}+1\left(\xi-\xi_{0}\right)
$$

F1rst, we note that Eqs. $(2,8)$ and $(2,9)$ can be approximated by. quadratures of the form [10]:

$$
\begin{aligned}
& \ddot{\tilde{I}}_{A} \propto \sum_{k=1}^{n} A_{k} \frac{f\left(\xi_{0}, n_{0}, \xi, n_{k}\right)}{n_{k}-n_{0}} u\left(\xi, n_{k}\right)-2 f\left(\xi_{0}, n_{0}, \xi\right) u\left(\xi, n_{0}\right) x_{n}\left(n_{0}\right) . \\
& \text { (1f } \left.n_{0} \neq n_{k}(k-1,2, \ldots, n)\right)
\end{aligned}
$$

and:

$$
f-\sum_{i=1}^{n} \Lambda_{k} \frac{f\left(\xi_{0}, n_{0}, \xi, n_{k}\right)}{n_{k}-n_{0}} u\left(\xi, n_{k}\right)+\left.\Lambda_{n} \frac{d\left[f\left(\xi_{0}, n_{0}, \xi, n\right) u(\xi, n)\right]}{d n}\right|_{n=n_{0}}-2 \varepsilon\left(\xi_{0}, n_{0}, \xi\right) u\left(\xi, n_{0}\right) \Lambda_{n}\left(n_{0}\right) \text {, }
$$

(if $\left.n_{0}=n_{m}(k=1,2, \ldots, n)\right)$,

with:

$$
R_{n}(t)=\frac{q_{n}(t)}{\sigma_{n}(t)},\left(n_{0}+n_{k}\right),
$$




$$
\Lambda_{n}(t)=\frac{1}{\sigma_{t}^{\prime}(t)}\left[q_{n}^{\prime}(t)+\frac{1}{4} A_{m} \sigma_{n}^{\prime \prime}(t)\right],\left(\eta_{0}=n_{m}\right)
$$

The functions $K_{n}(t), \Lambda_{n}(t)$ are systematically tabulated in ref.[12], according to the various numerical integration rules.

At the same time the principal value $I_{1}$ can be approximated by:

$I_{1} \simeq \sum_{\ell=1}^{m} A_{m} \frac{\left(\tilde{I}_{A}\left(\xi_{0}, n_{0}, \xi_{m}\right)-\tilde{I}_{B}\left(\xi_{0}, n_{0}, \xi_{m}\right)\right)}{\xi_{m}-\xi_{0}}-2\left(\tilde{I}_{A}\left(\xi_{0}, n_{0}\right)-\tilde{I}_{B}\left(\xi_{0}, n_{0}\right)\right) K_{n}\left(\xi_{0}\right)$,

where the functions $\tilde{\mathrm{I}}_{A}$, $\tilde{\mathrm{I}}_{B}$ are given by the expressions (2.11) and (2.12).

Let us consider now the speclal case where:

$w(\xi, n)=1$, (Gauss-Legendre numerical integration rule),

and :

$$
f\left(\xi_{0}, n_{0}, \theta\right)=\frac{\theta r}{\theta \xi}=\cos \theta .
$$

Then, the singular integral $I_{1}\left(\xi_{0}, n_{0}\right)$ becomes:

$$
I_{1}=\int_{T} \frac{\theta r / \theta \xi}{\left(\xi-\xi_{0}\right)^{2}+\left(n-n_{0}\right)^{2}} u(\xi, n) d \xi d n .
$$

Moreover, the quadrature $(2.15)$ may be rewritten as:

$$
I_{1} \simeq \int_{-1}^{1} \frac{\operatorname{Im}\left(I_{2}\left(\xi_{0}, n_{0}, n\right)\right)}{n-n_{0}} d n
$$

where $\operatorname{Im}\left(I_{2}\left(\xi_{0}, n_{0}, n\right)\right)$ denotes the imaginary part of the complex function:

$$
I_{2}\left(\xi, n_{0}, n\right)=\int_{-1}^{1} u(\xi, n) d(r / \xi-z)+\int_{-1}^{1} \frac{r}{(\xi-z)^{2}} u(\xi, n) d \xi \text {. }
$$

It is already well established [12] that, If $n_{0}$ is selected as a root of the Legendre function of the second kind and order given by:

$$
Q_{m}\left(n_{0}\right)=0 \text {, }
$$

a general method for the numerical evaluation of the integral $(2,18)$ can be read1ly established. This can be achieved by making one-dimensional numerical integrations (that is by using product rules [11]). Finally, it was concluded that:

$$
I_{1}\left(\xi_{0}, n_{0}\right)=\sum_{k=1}^{m} A_{k} \frac{I m\left(I_{2}\left(\xi_{0}, n_{0}, n_{k}\right)\right)}{\left(n_{k}-n_{0}\right)},
$$

where $n_{k}$ are the abscissas and $A_{k}$ the corresponding weights of the numerical 
Integration rule. Applying this, ic may be observed that the following improper Integral 18 needed to be calculated numerically:

$$
I_{3}\left(\xi_{0}, n_{0}\right)=\int_{-1}^{1} \frac{u(z, n) Q_{n}(z)}{P_{n}(z)\left|n-n_{0}\right|^{d n},}
$$

where $Q_{n}(z)$ denotes the Legendre function of the second kind and order $n$, whereas $P_{n}(z)$ expresses the Legendre polynomial of degree $n$. The existence of $I_{2}$ in an ordinary sense requires that:

$$
Q_{n}\left(\xi_{0}\right)=0 \text {, }
$$

where $n$ denotes the number of abscissas used for the numerical evaluation of the Integral $I_{2}$, and $\xi_{0}$ mot be selected as a root of the Legendre function of the second kind of order $n$.

Therefore, the pair of real numbers $\left(\xi_{0}, n_{0}\right)$, which are considered as a root of the Legendre function of the second kind of order $(n, m)$ respectively, defines a collocation point $I\left(\xi_{0}, n_{0}\right)$. The roots of the Legendre function of the second $k$ ind are oystenatically calculated and tabulated in ref.[10].

Finally it should be mentioned that, if the cubature formula $(2,22)$ is applied to a set of points $\left(\xi_{0}, n_{0}\right)$ different than the corresponding collocation points, the resulting numerical values should be invalid, and usually diverge. The case where $f\left(\xi_{0}, n_{0}, \theta\right)=\partial r / \partial n$ or $e^{1 n \theta}$ can be treated analogously.

3. APPLICATIOS OP THE CUBATURB FORAULAS

As an application of the above developwents the following two-dimensional Cauchy-type principal-value integral was evaluated:

$$
I_{4}=\int_{T} \frac{\cos \theta}{r^{2}} d s,
$$

where the variables are defined again by Bq.(2.2) and $T$ denotes the square with $-1<\xi, n<1$

On the basis of the results of Cruse [4], this integral was evaluated in a closed

$I_{4}=\ln \frac{\left.\left\{1-n_{0}+\left[\left(1+\xi_{0}\right)^{2}+\left(1-n_{0}\right)^{2}\right]^{1 / 2}\right\}-1-n_{0}+\left[\left(1-\xi_{0}\right)^{2}+\left(1+n_{0}\right)^{2}\right]^{1 / 2}\right\}}{\left\{-1-n_{0}+\left[\left(1+\xi_{0}\right)^{2}+\left(1+n_{0}\right)^{2}\right]^{1 / 2}\right\}\left\{1-n_{0}+\left[\left(1-\xi_{0}\right)^{2}+\left(1-n_{0}\right)^{2}\right]^{1 / 2}\right\}}$

On the other hand, the Integral $I_{4}$ was evaluated numerically by applying the above 
susgested Gauss-Legendre method. The results are given in Table II for several collocation points $x_{1}\left(\xi_{0}, n_{0}\right)$.

It is obvious from this table that, by increasing the orders ( $m \times n)$ of the Legendre polynomials, the values for $I_{4}$ converge fast and soothly towards their correspondins theoretical values derived on the basis of Eq. (3.2)

More precisely, in Table II, the numerically estimated values for $I_{4}\left(\xi_{0}, n_{0}\right)$ are given for an arbitrarily selected set of collocation points, that to for the points:

$$
\begin{aligned}
& x_{1}(0.3606231751,0.3606231751), \\
& x_{2}(0.3714922970,0.5403934260), \\
& x_{3}(0.5479477112,0.9509446082),
\end{aligned}
$$

The coordinates of the collocation points $X_{1}, I_{2}, X_{3}$ are selected in both cases to be the roots of the Legendre polynomials of the second kind of orders $=\mathrm{n}=8,16$ and 32 respectively [10].

Comparing the numerical values of the integral $I_{4}$ with their corresponding exact values given by relation (3.2), it may be implied that a rapid convergence exists. Indeed, the results presented in Table II seen to vconverge rapidly to their corresponding theoretical values, that 18, for the case where $8 \times 8$ absc1ssae are used, the values of $I_{4}$ was found to be within 29 percent of the exact value, while for the $16 \times 16$ case the discrepancy between these two values decreases to within 6 percent and finally, for the $32 \times 32$ case it becomes only 0.3 percent.

Finally, it 18 worth mentioning that the rate of convergence of the suggested numerical integration rule was mainly affected by the oscillatory behavior of the characteristic of the two-dimensional Cauchy principal-value Integral $I_{4}$. In the present case the characteristic function colncides with the function $\cos \theta$. 4. NUMERICAL EVALUATION OF 2-D PRINCIPAL VALUE INTEGRALS WITH A LOGARITHMC

\section{SINGULARITY}

The theory developed for the numerical evaluation of $2-D$ principal-value Integrals, containing an algebraic singularity, is extended to include the case of Cauchy principal-value Integrals Involving a Logarithmic singularity, Although such singular integrals are hardly found in physical problems, the derivation of a 
corresponding cubature formula is motivated by the fact that these integrals are a natural extension of the 2-D Cauchy principal-value integrals involving an algebraic singularity, extensively examined in the previous paragraph. We generalize now the cubature formula (2.15), which is valid for two-dimensional singular integrals, to singular integrals of the following form, presenting a logarithmic singularity:

$$
\begin{aligned}
I_{5}\left(x_{0}, y_{0}\right)= & f_{S} w_{0}(x, y) \frac{f_{0}\left(x_{0}, y_{0}, \vartheta\right)}{\left(x-x_{0}\right)^{2}+\left(y-y_{0}\right)^{2}} u_{0}(x, y) d S, \\
& \left(x_{0}, y_{0}\right) \in S,
\end{aligned}
$$

on a plane finite region $S$ (Fig.1), where,

$$
\left(x-x_{0}\right)+i\left(y-y_{0}\right)=r e^{1 \vartheta},
$$

while the logarithmic density function is expressed by

$$
\begin{aligned}
w_{0}(x, y)= & \frac{\sin ^{m}\left\{\left(x-x_{0}\right)^{2}+\left(y-y_{0}\right)^{2}\right\}}{\left\{\left(x-x_{0}\right)^{2}+\left(y-y_{0}\right)^{2}\right\}^{\lambda / 2}}, \\
& (m=1,2,3, \ldots, \ldots), \lambda \geq 1 .
\end{aligned}
$$

Moreover, $I_{5}\left(x_{0}, y_{0}\right)$ can be written as:

with:

$$
I_{5}=f_{S} w(r, \theta) \frac{f(\theta)}{r^{2}} u(r, \theta) d S,
$$

$f(\vartheta)=f_{0}\left(x_{0}, y_{0}, \vartheta\right), u(r, \vartheta)=u_{0}(x, y), w(x, \vartheta)=w_{0}(x, y) \ldots$

In the following 1 was assumed that the functions $u$ and $f$ satisfy the same conditions as previously stated (see Eq. $(2,4)$ ). On the other hand, the boundary of S (F1g.1) can be described by:

$$
R=R(\vartheta), \theta \in[0,2 \pi],
$$

where $R$ is considered as a single-valued, although the case of multivaluedness does not introduce any further considerable difficulties.

We shall now derive the cubature formula for the 2-D singular integral $I_{5}$ in accordance with relations $(4,4)$ to $(4.5)$ and reference [9]:

$$
I_{5}=\int_{0}^{2 \pi} f(\theta) d \theta f_{0}^{R(\theta)} w(r, \theta) \frac{u(r, \vartheta)}{r} d r .
$$

Equation (4.6) suggests the possibility of considering $I_{5}$ as a product of two one- 
dimensional integrals, by applying for the outer integral a product rule (1.e the trapezoldal rule with $L$ abscissas), while for the inner integral the already known finite-part integration quadratures [13]:

$$
\begin{aligned}
I_{6}= & \frac{2 \pi}{L} \sum_{1=0}^{L-1}\left(R\left(\frac{2 \pi 1}{L}\right)\right)^{1-\lambda}\left\{\sum_{j=0}^{8}\left(x_{j}\right) \ln (8-j)\left[R\left(\frac{2 \pi 1}{L}\right)\right]^{(s-j)}+\sum_{k=1}^{N} j_{k} u\left[R\left(\frac{2 \pi i}{L}\right)\right]\right]+ \\
& \left.+\frac{\ln (s+1)\left(R\left(\frac{2 \pi 1}{L}\right)\right)}{(s+1)(\lambda-1) !} \frac{d^{(\lambda-1)}\left[u\left(0, \frac{2 \pi 1}{L}\right)\right]}{d x^{(\lambda-1)}}\right\} .
\end{aligned}
$$

We observe that, if $\mathrm{jm}$ in the double sum, the corresponding weights ${ }_{0} \mathrm{w}_{k}$ are those of the quadrature formula for fintte-part integrals involving an algebratc singularity and wich are tabulated in [13]. The welghts $j_{k}$ for $j \neq 0$ are also included in [13]. Finally $x_{f}$ denote the equally spaced nodes $x_{j}=(j-1) / N,(j=1,2, \ldots, N)$.

As an application of the above developments the following 2-D Cauchy principalvalue integral was deteratned:

$$
I_{7}=\int_{S} w(\xi, n) \frac{\cos \theta}{r^{2}} u(\xi, n) d \xi d n
$$

where:

$$
w(\xi, n)=\ln (x) / x^{\lambda}, \quad\left(\xi-\xi_{0}\right)+1 n=r e^{1 \theta}
$$

The numerical results are presented in Table III for various positions $\xi_{0}$ of the second order pole $I\left(\xi_{0}, n_{0}\right)$ inside the unit disk $(F 1 g .2)$ with a weight function of the form (4,2), $(m-1, \lambda-1 / 2)$ or $(m-2, \lambda=1)$ and a density function $u(\xi, n)=\xi$ or $u(\xi, \eta)=\exp (\xi)$.

The total error of the cubature formula $(4,7)$ consists of one term depending upon the trapezoldal rule which is applied around the angle $\theta$ (the number of abscissas of this rule is denoted by the integer $L$ ), while the other one term is that of the finite-part integration rule, which is applied along the radius $r$ of the unit disk of $\mathrm{Fig.2}^{2}$ (the number of abscissas of this rule 1s denoted by the integer $\mathrm{N}$ ).

In order to check the rate of convergence of the proposed cubature (4.7), as L Increases, we consider the spectal ease of the density function $u(\xi, n)=\xi$ for which the error term belonging to the finite-part integration is zero (or equivalently independent or N) (Table III(a)). By observing thls Table we can see that as $\mathrm{L}$ 
increases from $L=18$ to $L=36$ or $L=72$ four additional digits of the numerical results are fixed (Independently of the values of the abscissa $N$ ): $\xi_{0}=0.2$ or 0.8 and ( -1 , $\lambda=1 / 2)$.

On the other hand, in order to check the rate of convergence of the cubature formula (4.7) as a function of the integer $N$, we consider the spectal case for which the density function $u(\xi, n)$ has the exponential form $\exp (\xi)$, while $I$ is taken to be large enough (1.e. $L \geq 72$ ) in order to minimize the trapezoldal error effects inherent to the numerical calculations (Table III(b)).

By observing this table we can see that as $N$ increases (from $N=3$ to 4 or 6 ), at least one additional digit of the numerical result is fixed $\xi_{0}=0.2$ or 0.6 and $(m=1, \lambda=1 / 2)$. The results given in table III are for the first time directly calculated for a two dimensional singular integral of the form (4.8), with a weight function different.:from unity, by applying a cubature formula of the form (4.7) for various positions of the second order pole $x\left(\xi_{0}, n_{0}\right)$ inside the untt disk $S(P 1 g .2)$. It should be also mentioned that the corresponding results referenced in the bibliography are those of Gabdulkhaev $[6,7]$ who succeeded in proposing a cubature formula for the singular integral $I_{7}$ in the special case where the weight function $w(\xi, n)=1$ and the second order pole $X\left(\xi_{0}, n_{0}\right)$ coincides with the :center of the unit disk $S$, that $18 \times\left(\xi_{0}, n_{0}\right)=x(0,0)$.

Furthermore, we can calculate a large variety of singular integrals of the form (4.8) by applying the cubature formula (4.7). As an example we can mention the cases $(m=2, \lambda=1)(L=54, N=8)$ for which $\left(\xi_{0}, I_{7}\right)=(0.2,61.442289)$ or $(0.4,74866826)$ or $(0.6,90.038705)$ or $(0.8,101.075295), u(\xi, n)=\exp (\xi)$. Another numerical example 1s: $(m=0, \lambda=0), u(\xi, n)=a \xi^{2}+b \xi^{2}+\gamma \xi^{2}+\delta \xi+\varepsilon, \quad(L=72, N=4), \quad\left(\xi, \alpha, \beta, \gamma, \delta, \varepsilon, I_{7}\right)=(0.2,0.5,0.5$ $1.5,1.0,1.0,-0.807914)$ or $(0.4,0.5,0.5,1.5,1.0,1.0,-4.080431)$ or $(0.6,0.5,0.5,1.5$, $1.0,1.0,-12.089886)$ or $(0.2,0.5,1.5,1.0,0.5,0.5,0.792218)$ etc. Another numerical example $18(m=1, \lambda=1 / 2) \quad(L=72, N=8), u(\xi, n)=a \xi+b, \quad\left(\xi, a, b, I_{7}\right)=(0.2,0.5,1,0$, $-6.2408940)$ or $(0.4,0.5,1.0,-5.9932751)$ or $(0.6,0.5,1.0,-5.0806756)$ etc.

As a conclusion, it can be claimed that a powerful numerical technique is developed for the numerical investigation of 2-D singular integrals of the form (4.8), defined over a planar region $S$ and presenting a logarithmic singularity of 
any type.

The proposed method seems to be new and can be considered as a direct generalization of several previous attempts to solve the simpler problem of the numerical calculation of the 2-D singular integral (4.8) for the special case $w(\xi, n)=1, \xi_{0}=0, \eta_{0}=0$, where S denotes the unit disk of Fig.2 (see: for example [3] to [8]).

5. MODIFIED GAUSS-LOBATTO AND GAUSS-RADAU CUBATURE FORMULAS

In problems of elasticity it is conventent to use cubature formulas where some of the abscissas are given in advance, whereas the others are free to be chosen according to some appropriate criterion. The case when a single station should be fixed in advance in the classical Gauss integration theory is generally called the Gouss-Radou integration, whereas the case when two preassigned stations are needed 18 called the Gauss-Lobatto rule.

In the following we shall develop appropriate integration rules for both modified types of integration. For this purpose we consider again the singular Integral (2.1). By Introducing a polar coordinate system with 1ts orfigin coinciding with the second-order pole of the singular integral (2.1) $x\left(\xi_{0}, n_{0}\right)$, we obtain that:

$$
\begin{aligned}
& f(\theta)=f\left(\xi_{0}, n_{0}, \theta\right), \\
& u(r, \theta)=u(\xi, n), \\
& d S=r d r d \theta .
\end{aligned}
$$

Based on Eq. (5.1) the principal value of the integral (2.1) way be written as:

$$
I_{8}=\lim _{\varepsilon \rightarrow 0} \int_{0}^{2 \pi} f(\theta) \int_{\varepsilon}^{R(\theta)} \frac{u(r, \theta)}{r} d r d \theta .
$$

where the weight function $w(\xi, \eta)$ equals unity, and $R(\vartheta)$ is defined by $\mathrm{Eq},(4,5)$.

Equation (5.2) suggests the posilbility of considering $I_{8}$ as a product of two one-dinensional integrals expressed by:

$$
I_{8}=\int_{0}^{2 \pi} \varphi(\theta) d \theta=\frac{2 \pi}{m} \sum_{1=0}^{m-1} \oplus\left(\frac{2 \pi 1}{m}\right),
$$

and:

$$
\varphi(\theta)=f(\theta) \int_{0}^{R(\theta)} \frac{u(r, \theta)}{r} d r .
$$

where the integral on the right-hand side of Eq. (5.4) may be defined in the finite- 
part sense [13].

As regards the numerical evaluation of the integrals of the form (5.4), it is valid that:

$$
\int_{0}^{R(\theta)} \frac{u(r, \theta)}{r} d r \propto \sum_{k=1}^{n} \hat{A}_{k} u\left(R(\theta) \rho_{k}, u\right)+u(0, \theta) \ln R(\theta) .
$$

where $p_{k}, A_{k}$ are the abscissas and the welghts respectively formally tabulated in ref.[13].

The previously developed cubature formula may be extended as follows to the Radau and Lobatto Integration rules;

1) In the case of Radou-type formula with the poin $x=0$ as the one preassigned station, the following one-dimenslonaz quadrature formula seems to be val1d $[13,9]$ :

$$
\int_{0}^{1} \frac{u(x)}{x} d x=\sum_{1=1}^{n} \frac{w_{1}^{*}}{x_{1}^{*}+1}\left[u\left(\frac{x_{1}^{*}+1}{2}\right)-u(0)\right] \text {. }
$$

where $w_{1}^{*}$ are the weights of the classical Gass-Legendre quadrature formula and $x_{1}^{*}$ denotes a zero of the Legendre polynomial or order $n$. By combining expressions $(5,3)$ and (5.6) together with a properly seleeted trapezoldal rule (with a abscissas), we obtain the following cubature formula for the numerical evaluationnof the singular Integral (5.2).

$$
\begin{gathered}
I_{8} \propto \frac{2 \pi}{n} \sum_{1=0}^{=-1} f\left(\frac{2 \pi 1}{m}\right)\left\{\sum _ { j = 1 } ^ { n } \frac { w _ { 1 } ^ { * } f } { x _ { j } ^ { * } + 1 } \left[u\left(\frac{x_{j}^{*}+1}{2}, \frac{2 \pi 1}{m}\right)-\right.\right. \\
\left.\left.-u\left(0, \frac{2 \pi 1}{m}\right)\right]+u\left(0, \frac{2 \pi 1}{m}\right) \ln \left[\mathrm{R}\left(\frac{2 \pi 1}{m}\right)\right]\right\},
\end{gathered}
$$

where $x_{j}^{*}$ are 1dentical with the zeros of the Legendre polynomials of order $n$, while $w_{j}^{*}$ are the weights of the classical Gauss-Legendre.quadrature formula.

11) The second Important case of the Lobatto type quddrature, with the points $x=0,1$ as preaseigned stations, way be treated sinilarly. For this case It 1s valld that [13]:

$\int_{0}^{1} \frac{u(x)}{x} d x \propto \sum_{1=1}^{n} \frac{w_{1}}{1-x_{1}^{2}}\left[\frac{x_{1}-1}{2} u(0)-\frac{x_{1}+1}{2} u(1)+u\left(\frac{x_{1}+1}{2}\right)\right]-u(0)+u(1)$,

where $w_{1}, x_{1}$ are the welghts and the stations, derived from the special Jacobi 
polynomials $\mathrm{P}^{(1,0)}(\mathrm{x})$. These coefficients are systematically tabulated in ref $:[14]$.

By properly combining expressions (5.5) and (5.8), we may conclude that, for the case of the Lobatto-type integration rule, the following modified cubature formula is valid:

$$
\begin{aligned}
I_{8} \simeq & \frac{2 \pi}{m} \sum_{i=0}^{m-1} f\left(\frac{2 \pi i}{m}\right)\left\{\sum _ { j = 1 } ^ { n } \frac { w _ { j } } { 1 - x _ { j } } \left\{\frac{x_{j}-1}{2} u\left(0, \frac{2 \pi i}{m}\right)-\frac{x_{j}+1}{2} u\left(1, \frac{2 \pi i}{m}\right)+\right.\right. \\
& \left.\left.+u\left(\frac{x_{j}+1}{2}, \frac{2 \pi i}{m}\right)\right\}+u\left(1, \frac{2 \pi i}{m}\right)+u\left(0, \frac{2 \pi i}{m}\right)\left[\ln \left[R\left(\frac{2 \pi i}{m}\right)\right]-1\right]\right\} .
\end{aligned}
$$

6. ERROR ESTTMATES FOR 2-DIMENSIONAL PRINCIPAL VALUE INTEGRALS

An error estimate is derived for the cubature formula, which was proposed in the previous paragraph (Eqs. $(4,6)$ or $(5,2)$ ), and the convergence of this formula is established.

Let us consider now the two-dimensional singular integral of the form:

$$
H=\int_{0}^{2 \pi} w(\vartheta) f(\vartheta) H_{A}(\vartheta) d \vartheta \text {, }
$$

where:

$$
H_{A}(\vartheta)=\int_{0}^{1} w(r) \frac{u(r, \vartheta)}{r} d r+p(n) \frac{u^{(n-1)}(0, \vartheta)}{(n-1) !} \times l n|R(\vartheta)| \text {, }
$$

with $w(r)=r^{1-q}$ and:

$$
p(n)=\left\{\begin{array}{l}
1, \text { if } q \text { is an integer } \\
0, \text { if } q \text { is a rational number. }
\end{array}\right.
$$

Here the variables $r$ and $\vartheta$ have been already introduced in relation $(4,2)$, while the functions $u(r, \theta), f(\theta)$ satisfy the conditions $(2,4)$, and $R(\theta)$ is given again by $\mathrm{Eq} \cdot(4,5)$.

Next, we can assume that the function $u(z, \vartheta)$ is an analytic function of $z$, regular in the disk $|z|<\varepsilon$, which contains the integration interval $[0,1]$; $1, e$,

$$
u(\vec{z}, \vartheta)=\sum_{k=0}^{\infty} b_{k} z^{k},
$$

with:

$$
b_{k}=\frac{u^{(k)}(0, \vartheta)}{k !} .
$$

Then, according to the developments of Hardy ([15] p.83), the integral:

$$
H_{B}=\int_{0}^{\infty} e^{-t} F(z t) d t \text {, }
$$


where:

$$
F(z)=\sum_{k=0}^{\infty} h_{k} \frac{z^{k}}{\Gamma(1+k)},
$$

represents a radial continuation of the function $u(r, \vartheta)$ on a definite region of summability. This practically means that the function $u(z, \vartheta)$ has the form:

$$
u(z, \vartheta)=\int_{0}^{\infty} e^{-t}\left[\sum_{k=0}^{\infty} b_{k} \frac{(t z)^{k}}{k !}\right] d t .
$$

Then, the error term can be calculated as follows:

$$
\left|R_{n}(u)\right|=\left|\int_{0}^{1} w(r) \frac{u(r, \vartheta)}{r} d r-\sum_{k=1}^{n} w_{k} u\left(r_{k}, \vartheta\right)\right|=\mid \int_{0}^{\infty} e^{-t} \sum_{k=0}^{\infty} \frac{t^{n+k}{ }^{n+k}}{(n+k) !} c, k d t \text {, }
$$

with:

$$
c_{n, k}=\int_{0}^{1} w(r) r^{n+k-1} d r-\sum_{k=1}^{n} w_{k} x_{k}^{n+k} .
$$

Furthermore, it follows from Eq.(6.9) that:

$$
\left|R_{n}(u)\right| \leqq \int_{0}^{\infty} e^{-t} \sum_{k=0}^{\infty}\left|\frac{t^{n+k} b_{n+k}}{(n+k) !} n, k\right| d t,
$$

or, after applying Hölder's inequality to Eq. $(6.11)$, we obtain:

$$
\left|R_{n}(u)\right| \leqq \int_{0}^{\infty} e^{-t}\left[\sum_{k=0}^{\infty}\left|\frac{t^{n+k} b_{n+k}}{(n+k) !}\right|^{q}\right]^{1 / q}\left[\left.\sum_{k=0}^{\infty} i c_{n, k}\right|^{p}\right]^{1 / q} d t \text {. }
$$

with:

$$
1 / p+1 / q=1
$$

Thus, the error bound can be written as:

$$
\left|R_{n}(u)\right| \leq \psi_{1}(n, p) \Psi_{2}(u, q)
$$

where:

$$
\psi_{1}(n, p)=\left[\sum_{k=0}^{\infty}\left|c_{n, k}\right| p\right]^{1 / q}
$$

and

$$
\psi_{2}(u, q)=\int_{0}^{\infty} e^{-t}\left[\left.\sum_{k=0}^{\infty}\left|\frac{t^{n+k} b_{n+k}}{(n+k) !}\right|\right|^{p / q}\right.
$$

We shall now consider the following important cases:

i) The case of $\psi_{1}(n, 2), \psi_{2}(u, 2)$. that is the fuclidean norm of $\psi$, for which it is valid that: 


$$
\psi_{1}^{2}(n, 2)=\sum_{k=0}^{\infty} c_{n, k}^{2}
$$

1i) The case $\psi_{1}(n, \infty), \psi_{2}(u, 1)$ will be examined next, by which the supremum norm may be obtained, that is:

$$
\psi_{1}(n, \infty)=\sup _{k=0,1, \ldots}\left|c_{n, k}\right|
$$

The main problem of the present analysis concerns the conditions of existence of the functions $\psi_{1}(n, z)$ and $\psi_{1}(n, \infty)$.

In order to prove the existence of the function $\psi_{1}(n, z)$, we investigate the series $\sum_{k} c_{n, k}^{2}$. We can rewrite Eq. $(6.16)$ in the form:

$$
\psi_{1}^{2}(n, 2)=\sum_{k=0}^{\infty} L_{n+k}-2 \sum_{k=0}^{\infty} L_{n+k} S+\sum_{k=0}^{\infty} S_{k}^{2} \text {, }
$$

with:

$$
\begin{aligned}
& L_{n+k}=\int_{0}^{1} x^{n+k-\lambda} d x, \\
& s_{k}=\sum_{i=1}^{n} w_{i} x_{1}^{n+k} .
\end{aligned}
$$

Since it is valid that:

$$
\left|s_{k}\right|<\sum_{i=1}^{n}\left|w_{i}\right| x_{i}^{n+k}<\left[\sum_{i=1}^{n}\left|w_{i}\right|\right]\left(\max x_{i}\right)^{n+k}
$$

which means that the series $\left[\sum_{i=1}^{n}\left|w_{i}\right|\left(\operatorname{maxx}_{i}\right)^{n+k}\right]$ is a convergent majorant series for $\sum_{k} S_{k}$, then it can be concluded that the second and third series in Eq. (6.21) are absolutely convergent. On the other hand, the first series on the right-hand side in Eq. (6.18) represents a general harmonic series, which converges absolutely. Therefore the first, second and third series in Eq. (6.18) are absolutely convergent and as consequence, $\psi_{1}(n, 2)$ exists. On the other hand, the function $\psi_{1}$ can be calculated numerically by simply applying Eq. (6.18).

As an example we have that $\left(n=4, \psi_{1}(n, 2)=0,289\right),\left(n=6, \psi_{1}=0,223\right),\left(n=8, \psi_{1}=0,186\right)$, $\left(n=10, \psi_{1}=0,162\right),\left(n=12, \psi_{1}=0,145\right),\left(n=14, \psi_{1}=0,132\right)$ are the calculated values of the factor $\psi_{1}$.

By properly combining expressions (6.1) to (6.3), we obtain that:

$$
H=\int_{0}^{2 \pi} \varphi_{1}(\vartheta) d \vartheta+\int_{0}^{2 \pi} \varphi_{2}(\vartheta) d \vartheta \cdot r \int_{0}^{2 \pi} \varphi_{3}(\vartheta) d \vartheta,
$$


where:

$$
\begin{aligned}
& \varphi_{1}(\vartheta)=\sum_{i=1}^{n} w_{i} u\left(r_{i}, \vartheta\right), \\
& \varphi_{2}(\vartheta)=R_{n}(u, \vartheta), \\
& \varphi_{3}(\vartheta)=p(n) \frac{u^{(n-1)}(0, \vartheta)}{(n-1) !} \ln |R(\vartheta)| .
\end{aligned}
$$

Here the quantities $w_{i}, r_{i}, R_{n}, p(n), R(\vartheta)$ are defined by relations $(4.2),(4.5)$ and (6.13). As a conclusion, the total error of the cubature formula (6.1) is given by:

$$
R_{t}(u, f)=R_{n}\left(u, \varphi_{1}\right)+R_{n}\left(u, \varphi_{2}\right)+R_{n}\left(u, \varphi_{3}\right) \text {, }
$$

where $R_{n}\left(u, \varphi_{1}\right)$ is the error expression of the trapezoidal rule for the finite integration interval $[0,2 \pi]$.

In order to determine the error $R_{n}\left(u, \varphi_{1}\right)$, we consider the function $\psi(z)$ defined by :

$$
\psi(z)= \begin{cases}\pi \exp \{+i \pi[v(z-a)-\lambda], & I_{m} z>0 \\ \pi \exp \{-1 \pi[v(z-a)-\lambda], & I_{m} z<0,\end{cases}
$$

where a, $\nu$ and $\lambda$ are real constants, $\lambda$ being such that $0 \leq \lambda<1$. Because of the fact that this function is analytic in each half-plane, we obtain, according to the developments of Donalson and Elliott [16], the relation:

$$
R_{n}\left(u, \varphi_{1}\right)=\frac{1}{2 \pi 1} \int_{C} \frac{\psi(z)}{\mu(z)} \varphi_{1}(z) d z,
$$

where $\psi(z)$ is given by the relation $(6,27)$ and $\mu(z)$ is defined by:

$$
\mu(z)=-\sin \pi[v(z-a)-\lambda] \text {, }
$$

Moreover the functions $\varphi_{1}(z)$ are defined by Eqs, $(6.23)$ to $(6.25)$. The curve $C$, which surrounds the interval $[0,2 \pi]$, is defined in ref.[16] (p.584).

The integrals of the form $(6,28)$ can be calculated directly and, because of the analyticity of their integrands, they are finite and bounded. Therefore, by applying Eq. (6.26), the error of the cubature formula (4.7) follows as a definite and bounded quantity. 


\section{i. CONCLUSIONS}

In the present paper the general method, already introduced in refs.[1] and [2], s extended to include the cases of the Lobatto- and Radau-integration rules, for the alculation of 2-D singular integrals. This construction of the cubature formulas of e Gaussian type has the following advantages, in comparison with the approximate tecrniques of Cruse et a1.[3]:

i) The density function $u(r, \vartheta)$ is no longer approximated by an algebraic polynomial $\left(a x+b\right.$ or $\left.a x^{2}+b x+c(a, b, c=c o n s t a n t s)\right)$, in order to calculate the integrals or to solve the 2-D singular integral equations resulting from physical problems under consideration.

ii) The range of integration $\mathrm{S}$ is no longer divided into small triangles etc in order to calculate the C.P.V. of the 2-D integrals.

iii) It permits for the first time, the calculation of $\sqrt{ } 2-D$ singular integrals with a weight function $w(r, \vartheta)$ different from unity.

iv) The error quantities and the proof of convergence of the resulting Gaussian cubature formulas is established.

v) The concept of collocation points is extended to three-dimensional problems by using the techniques of complex analysis [7].

As a consequence, correct numerical values are found for certain 3-D problems with a much smaller computer-time requirement.

vi) The numerical technique developed in this paper is simpler and less timeconsuming method than the BIE-method, since it does not need to discretize the surface into small pieces, on which the integration must be done while the surface data of the density function do not need to be considered as constant. This is an advantage of the suggested method as compared with the BIE-method $[4,6]$. vi1) The method developed can be easily extended to solve numerically systems of two-dimensional singular integral equations [10]. From this point of view it seems to be the two-dimensional counterpart of the already established theory for the numerical solution of one-dimensional singular integral equations. ACKNOWLEDGFMENT. Author wishes to thank Mr. J. G. Kazantzakis for his help during the preparation of the paper. 


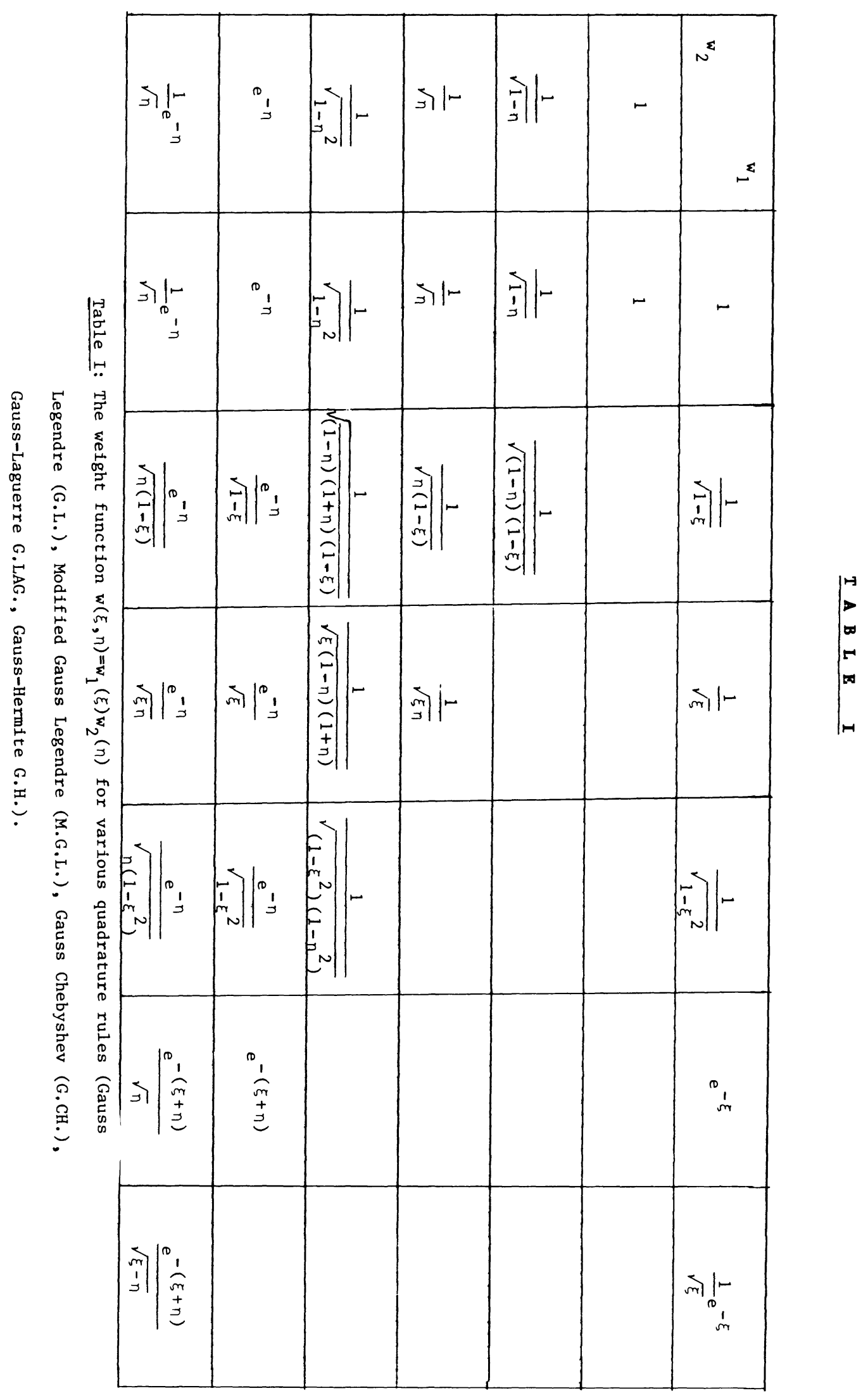


\begin{tabular}{lllllll}
$\mathbf{I}$ & $\mathbf{A}$ & $\mathbf{B}$ & $\mathbf{L}$ & $\mathbf{B}$ & $\mathbf{I} \mathbf{I}$ \\
\hline
\end{tabular}

\begin{tabular}{|c|c|c|c|}
\hline$(m \times n)$ & $\begin{array}{l}\text { Collocation Points } \\
x_{1}\left(\xi_{0}, n_{0}\right) 1=1,2,3\end{array}$ & $\begin{array}{l}\text { Numerlcal } \\
\text { value of I } \\
(\text { Eq. (22)) }\end{array}$ & $\begin{array}{l}\text { Theoretical } \\
\text { value of I } \\
(E q \cdot(26))^{4}\end{array}$ \\
\hline$(8 \times 8)$ & $\begin{array}{r}x_{1}(0.3606231751 \\
0.3606231751)\end{array}$ & -1.342879 & -1.045333 \\
\hline$(16 \times 16)$ & $\begin{array}{r}x_{2}(0.3714922970 \\
0.54039 \\
34260)\end{array}$ & -1.069729 & -1.011953 \\
\hline$(32 \times 32)$ & 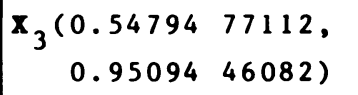 & -1.194753 & -1.191160 \\
\hline
\end{tabular}

Table II: Numerical and theoretical values of the integral $I_{3}-\int_{S} \frac{\cos \vartheta}{2} d S$ for $m \times n=(8 \times 8)$, $(16 \times 16)$ and $(32 \times 32)$.

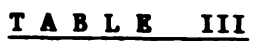

\begin{tabular}{|c|c|c|}
\hline \multicolumn{3}{|c|}{$(\alpha)$} \\
\hline$\xi_{0} 0$ & 0.2 & 0.8 \\
\hline 18 & -12.5143188 & -8.7161754 \\
\hline 36 & -12.5147725 & -8.7161762 \\
\hline 72 & -12.5147725 & -8.7161762 \\
\hline
\end{tabular}

\begin{tabular}{|c|c|c|}
\hline \multicolumn{3}{|c|}{$(B)$} \\
\hline$L$ & 0.2 & 0.6 \\
\hline 3 & -15.0669552 & -20.1688455 \\
\hline 4 & -15.3306996 & -20.5379592 \\
\hline 6 & -15.3382977 & -20.5853835 \\
\hline
\end{tabular}

Table III: (a) Numerical evaluation of the 2-D singular integral I, (eqn.34) for the case where $(m=1, \lambda=1 / 2)$ and $u(\xi, n)=\xi$;

(b) Numerical evaluation of the 2-D singular integral I (eqn.34) for the case where $(m=1, \lambda=1 / 2, u(\xi, n)=\exp \xi$ and $L=72$. 


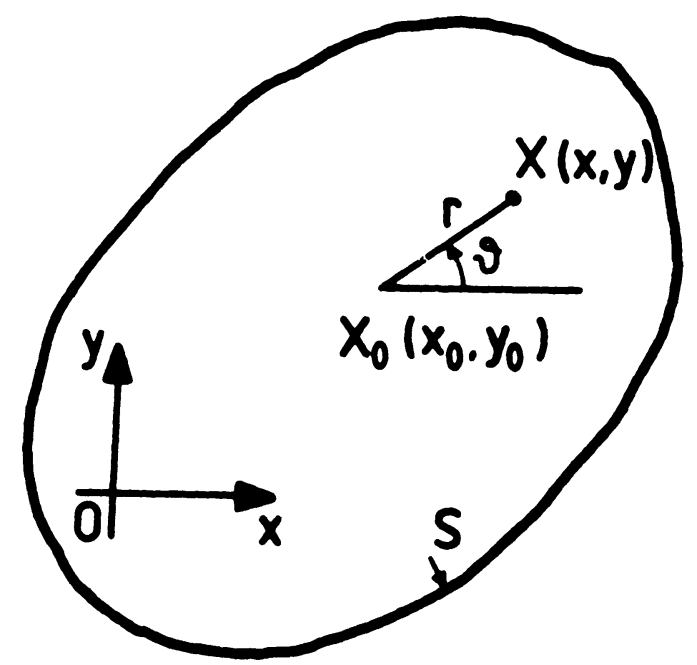

Fig. 1. Geometry of the two-dimensional region.

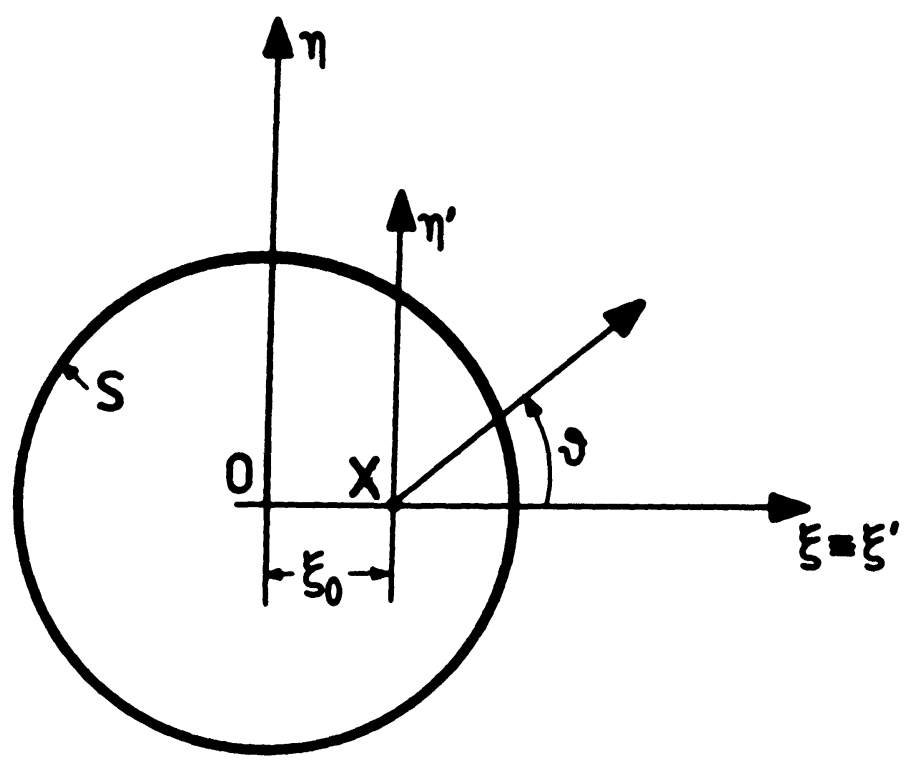

Fig. 2. Geometry of a unit disk $S$ with a second-order pole $X\left(\xi_{0}, n_{0}\right)$ inside.it. 


\section{REFERENCES}

1. KAZANTZAKIS, J,G. and THEOCARIS, P.S. On the evaluation of certain two-dimensional singular integrals used in three-dimensional elasticity, Int. J. Solids Struct., 15 (1979), 203-207.

2. TSAMASPHYROS, G. and THEOCARIS, P.S. Cubature formulas for the evaluation of surface singular integrals, Nord. Tidsk. for Inform. Behand. (BIT), 19 (1979), 368-377.

3. CRUSE, T.A. Numerical solutions in three-dimensional elastostatics, Int. J. Solids Struct. , $\underline{5}$ (1969), 1259-1274.

4. CRUSE, T.A. Application of the boundary-integral equation method to threedimensional stress analysis, Comp. and Struct., 3 (1973), 509-527.

5. CRUSE, T.A. and WILSON, R.B. Advanced applications of boundary-integral equation methods, Nucl. Engng. Des., 46 (1978), 223-234.

6. GABDULKHAEV, B.G. Cubature formulas for multidimensional singular integrals, II, IZV. Vys8. Uch. Zav. Matem., 4 (1975), 3-13 [English translation in the corresponding issue of Sov. Math. (IZv. VUZ)].

7. GABDULKHAEV, B.G. and ONEZOV, L.A. Cubature formulas for singular integrals, Izv. Vys8. Uch. Zav. Matem., 7 (1976), 100-105 [English translation in the corresponding issue of Sov. Math. (Izv, VUZ)].

8. RIZZO, F.J. and SHIPPY, D.J. An advanced boundary integral equation method for three-dimensional thermoelasticity, Int. J. Num. Meth. Engng., 11 (1977), 1753-1768.

9. THEOCARIS, P.S., IOAKIMIDIS, N.I. and KAZANTZAKIS, J.G. On the numerical evaluation of two-dimensional principal value integrals, Int. J. Num. Meth. Engng., 15 (1980), 629-633.

10. THEOCARIS, P.S. and IOAKIMIDIS, N.I. Tables of collocation points for the numerical solution of cauchy-type singular integral equations, Publications of National Technical University, Athens, 1978.

11. TRICOMI, F. Equazioni integrali contenenti 11 valor principale di un integrale doppio (Integral equations containing the principal value of a double integra1), Math. Zeit., 27 (1928), 87-133.

12. THEOCARIS, P.S. and IOAKIMIDIS, N.I. Numerical integration methods for the solution of singular integral equations, Quart. Appl. Math., 35 (1977), 173-183.

13. KUTT, H.R. On the numerical evaluation of principal value integrals by finitepart integration, Special report No. WISK 179, The National Research Institute for Mathematical Sciences, Pretoria, 1975 [Ava1lable also from NTIS].

14. KRYLOV, V.I., LUGIN, V.V., YANOVICH, Tables for numerical integration of functions with power singularities, Minsk, Izdat. Akad. Nauk. BSSR, 1963.

15. HARDY, G.H. Divergent series, Oxford University Press, London, 1949.

16. DONALSON, J.D. and ELLIOTT, DAVID, A unified approach to quadrature rules with asymptotic estimates of their remainders, SIAM J. Numer. Anal. $\underline{9}$ (1972), 513-602. 


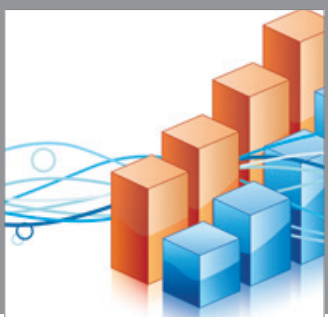

Advances in

Operations Research

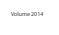

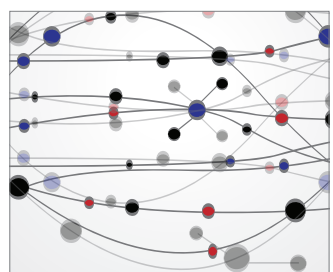

\section{The Scientific} World Journal
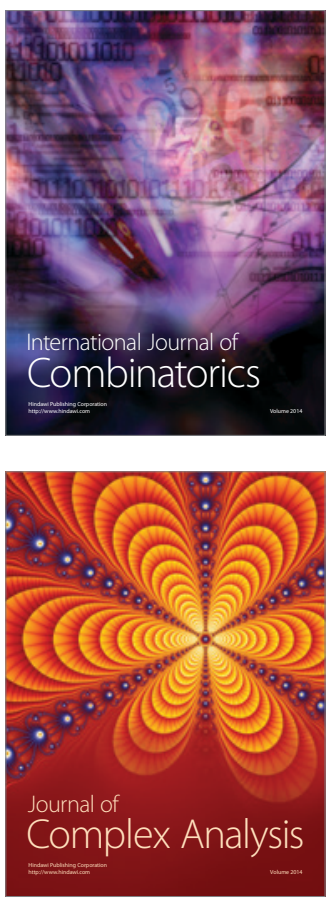

International Journal of

Mathematics and

Mathematical

Sciences
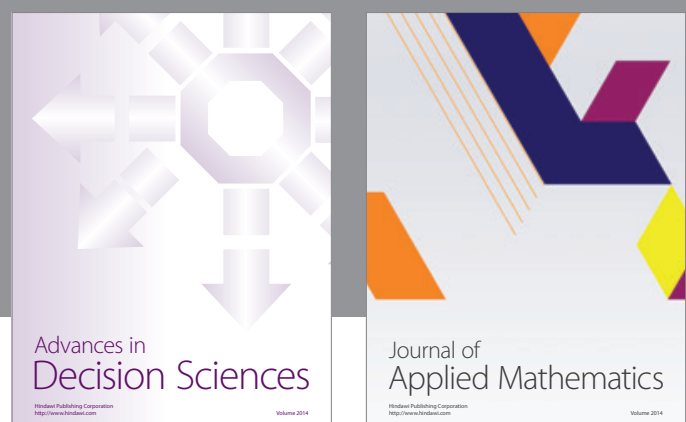

Journal of

Applied Mathematics
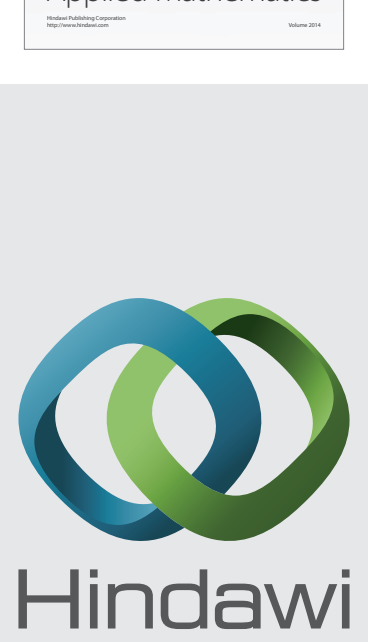

Submit your manuscripts at http://www.hindawi.com
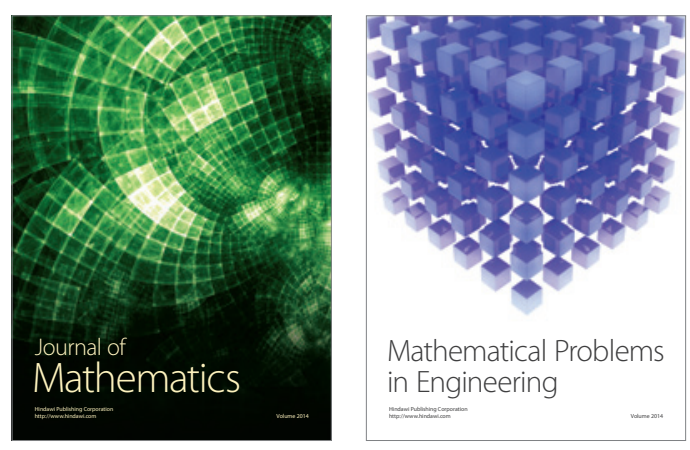

Mathematical Problems in Engineering
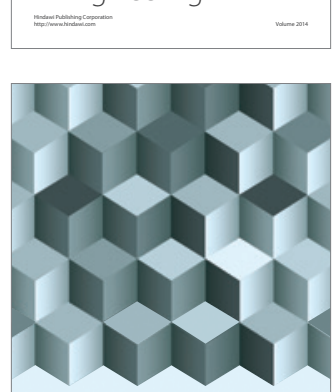

Journal of

Function Spaces
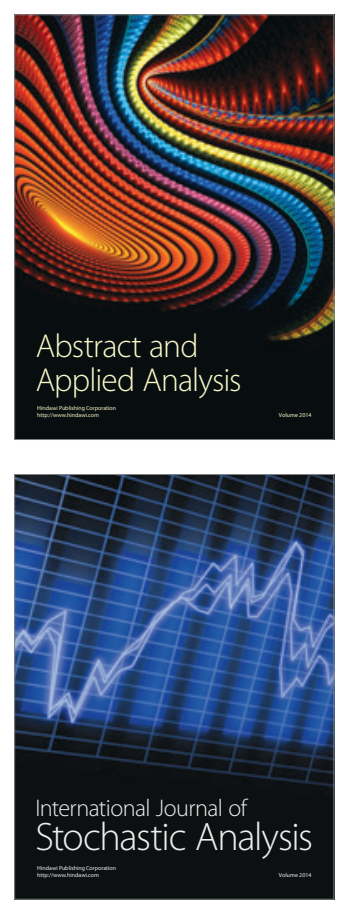

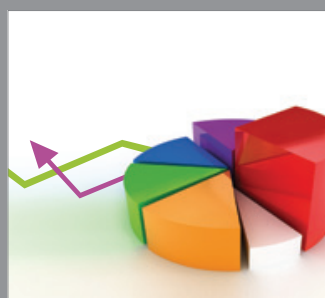

ournal of

Probability and Statistics

Promensencen
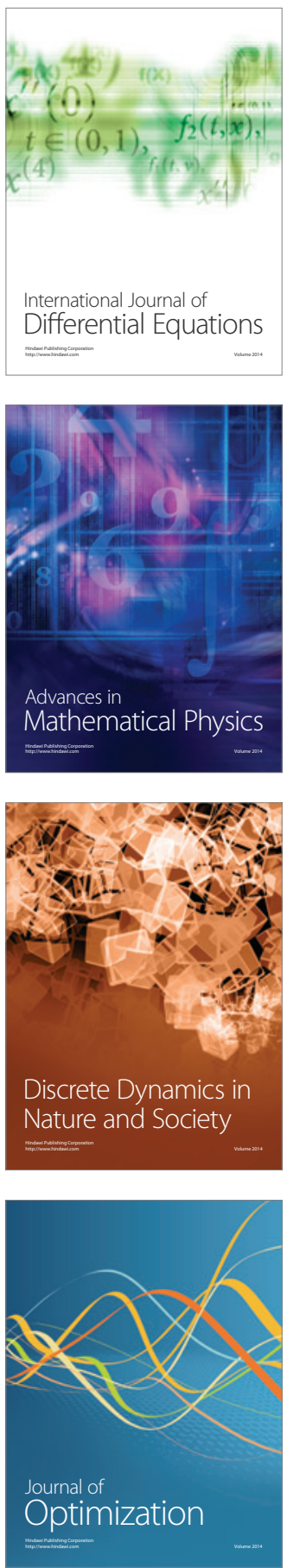